\title{
ALGUNOS ASPECTOS SOBRE LA INTRODUCCIÓN DE ESPECIES, Y ESTADO DEL CONOCIMIENTO SOBRE LOS PECES INTRODUCIDOS EN EL DEPARTAMENTO DE CALDAS, COLOMBIA.
}

\author{
DANIEL RESTREPO-SANTAMARÍA ${ }^{1}$ \\ RICARDO ÁLVAREZ-LEÓN ${ }^{2}$
}

Recibido el 24 de mayo de 2011 y aprobado el 5 de abril de 2013

\begin{abstract}
RESUMEN
La introducción de especies de peces ha sido considerada como uno de los problemas más difíciles de conservación de la biodiversidad, ya que la degradación de los ecosistemas naturales que ha venido ocurriendo en todo el mundo ha provocado que las especies no nativas se establezcan y se conviertan en invasoras con mayor facilidad. Objetivo: dada la importancia de la introducción de especies de peces y el papel que desempeñan sobre las especies nativas, realizamos una revisión bibliográfica, donde introducimos al lector a cerca del concepto de introducción de especies, algunos aspectos en la introducción de peces no nativos, describimos el conocimiento actual de las especies de peces introducidos en Colombia, y por último determinamos el estado actual de conocimiento sobre las especies de peces introducidas en los ecosistemas fluviales del departamento de Caldas. Metodología: Se realizo un compendio de información con base en artículos, libros e informes técnicos, acerca de la introducción de peces en el departamento de Caldas .Resultados: se registran 18 especies introducidas en aguas naturales de carácter invasor y 72 introducidas en cuerpos de agua artificiales (acuarios y estaques). Del total de especies no nativas 33 pertenecen al orden Perciformes, seguido de los Cypriniformes (12 especies), Characiformes (8 especies) y Cyprinodontiformes (8 especies). Las familias con mayor número de especies no nativas corresponden en su orden: Cichlidae con 24, Cyprinidae con 12 y Poeciliidae con ocho. Conclusiones: la permanencia de estas especies en el departamento de Caldas puede desencadenar efectos en los ecosistemas fluviales, por lo que hay que aplicar normas preventivas para llevar a cabo introducciones de especies en la región.
\end{abstract}

\section{PALABRAS CLAVE}

Invasión biológica, introducción de especies, trasplante, peces, Colombia.

\section{SOME ASPECTS ABOUT THE INTRODUCTION OF SPECIES, AND STATE OF KNOWLEDGE ON THE NON-NATIVE FISH IN THE DEPARTMENT OF CALDAS, COLOMBIA}

\begin{abstract}
The introduction of fish species has been considered as one of the most difficult issues of biodiversity conservation since the degradation of natural ecosystems which has been occurring all around the world has caused that non- native species establish themselves and easily become invaders. Objective: Because of the importance of introducing fish species and their role in native species, a bibliographic review was carried out in which the reader gets introduced to the concept of introduction of species, some
\end{abstract}


aspects of introduction of non- native fish species, describing the current knowledge about the introduced species of fish in Colombia, and finally, the present state of the knowledge of introduced species in the fluvial ecosystems of the department of Caldas is determined. Methodology: A summary of information based on articles, books and technical reports dealing with the introduction of fish in the department of Caldas was carried out. Results: Eighteen species were registered as introduced in natural water as invaders and 72 were registered as introduced in artificial water tanks (aquariums and ponds). From the total of non- native species, 33 belong to the Perciformes order, 12 to Cypriniformes order, 8 species to Characiformes order and 8 species to Cyprinodontiformes order. The families with the greatest number of non-native species are: Cichlidae (24 species), Cyprinidae (12 species) and Poeciliidae (8 species). Conclusions: the permanence of these species in the department of Caldas can trigger effects in the fluvial ecosystems, reason why it is necessary to apply preventive regulations in order to carry out introduction of species in this region.

\section{KEY WORDS}

Biological invasion, introduction of species, transplantation, fish, Colombia.

\section{INTRODUCCIÓN}

El Convenio sobre la Diversidad Biológica (CDB) ha sido adoptado por muchos países, lo que resulta en el desarrollo de estrategias nacionales de biodiversidad. Además, pone de manifiesto el reconocimiento internacional de la importancia de proteger los ecosistemas(PNGIBSE, 2012). Sin embargo, estos todavía se enfrentan a muchas amenazas, algunas de ellas crecen y se extienden tan rápidamente como para causar deterioro irreversible en muchos países y áreas (Urano, 2006). Los cambios en los ecosistemas han sido atribuidos principalmente a la pérdida de hábitat, la sobreexplotación de los recursos de los ecosistemas, la invasión biológica, la contaminación de nutrientes, la contaminación química tóxica y el cambio climático global (Urano, 2006), dando como resultado que muchas plantas y animales nativos de un ecosistema en particular pierdan sus espacios de vida. Estos cambios en los ecosistemas naturales en todo el mundo, han provocado que las especies introducidas se establezcan y se conviertan en invasoras con mayor facilidad (Elvira, 2005).

Se define una especie introducida como cualquier especie ajena (especie no nativa) al sitio que ha llegado, la cual es procedente de otro sitio de origen. Esta es liberada intencional o accidentalmente por el hombre; además, esta definición es utilizada en varios países para diferentes grupos de organismos. Sin embargo, desde un punto de vista ecológico, independientemente del origen geográfico, la introducción es entendida como la inserción de un elemento totalmente nuevo en una región dada (Delariva y Agostinho, 1999; Sarmiento, 2000; Gutiérrez-Bonilla, 2012).

Es probable que la introducción de especies no nativas en un ecosistema siempre presente un riesgo ecológico si la especie es capaz de integrarse con éxito al medio (Granado-Lorencio, 2000; Gozlan \& Newton, 2009). Esto da lugar a posibles interacciones perjudiciales con especies nativas 0 incluso en el funcionamiento del ecosistema (Gozlan et al., 2010); es decir, 
estas interacciones pueden afectar a la biodiversidad a través de la depredación (incluyendo herbivoría), competición, hibridación, uso de hábitat, reproducción, crianza y transmisión de enfermedades (Simberloff, 1996; Begon et al., 2006; Gutiérrez-Bonilla, 2006; Gozlan et al., 2010). Courtenay (1993) instauró la expresión "contaminación biológica" para referirse a estas acciones. De este modo, las especies exóticas invasoras se convierten en el segundo factor de riesgo de pérdida de diversidad biológica quedando detrás de la destrucción de hábitat (Moyle y Leidy, 1992; Allan y Flecker, 1993; Elvira et al., 2007).

Los impactos de las especies no nativas se ven reflejados sobre la fauna nativa, especialmente si adquieren el carácter de invasoras, debido a que proliferan a costa de las autóctonas (Baptisteet al., 2010) Esto conlleva, a muchos conservacionistas e investigadores, a creer que las invasiones y extinciones están estrechamente vinculadas (Gurevitchy Padilla, 2004.). Se estima que el $39 \%$ de las extinciones conocidas de animales desde el siglo XVI, se deben a la introducción de especies (Lodge, 1993). Además, la introducción de especies no nativas invasoras puede alterar el equilibrio de los ecosistemas locales o incluso destruirlo, debido a la ausencia de competidores o enemigos naturales (Urano,2006).Muchas de estas especies no nativas invasoras son hábiles colonizadoras que toman ventaja de la limitada competencia, y suelen ser resistentes a la degradación de los hábitats (Elvira, 2005).

\section{ALGUNAS CONSIDERACIONES SOBRE LA INTRODUCCIÓN DE PECES}

Las especies de peces representan el grupo de animales acuáticos más introducidos en el mundo (624 especies); además, son uno de los más amenazados (3120 especies de peces dulceacuícolas en el año 2009). Estas introducciones pueden deberse a la demanda social de productos pesqueros para la alimentación acuícola (51\% de las introducciones), peces ornamentales (21\%), pesca deportiva (12\%) y la pesca artesanal (7\%) (Gozlan, 2008; Gozlan y Newton, 2009; IUCN,2009). Cabe mencionar que no todos los peces introducidos se establecen, y la fracción de los que lo hacen a menudo tiene pocos efectos apreciables sobre sus nuevos ecosistemas, muchos otros ejercen significativos impactos ecológicos, evolutivos, y económicos (Cucherousset y Olden, 2011).

Sin embargo, las especies de peces no nativas y de carácter invasor son cada vez más reconocidas como un importante contribuyente a la amenaza de extinción de especies en aguas dulces, ya que este factor se une y combina con la pérdida y fragmentación del hábitat, alteración hidrológica, el cambio climático, la sobreexplotación y la contaminación (Dudgeon et al., 2006). Adicionalmente, los peces invasores afectan directa e indirectamente a los organismos nativos, desde el zooplancton hasta los mamíferos, a través de diversos niveles de organización biológica, desde el genoma hasta el ecosistema (Gozlan y Newton, 2009).

Una información más detallada a cerca de los efectos negativos de la introducción de especies de peces no nativos y las interacciones ecológicas que se dan en las comunidades naturales, se puede encontrar en los trabajos de Moyle y Light (1996), Agostinho et al. (2005), Gozlan et al. (2010) y Cucherousset y Olden (2011), estos proveen un panorama de la 
literatura más reciente sobre impactos ecológicos asociados a los aspectos de la introducción de especies de peces no nativos, e incluye cuestiones relacionadas con las vías de introducción, definiciones, los impactos ecológicos y económicos, evaluaciones de riesgo, y opciones de gestión.

\section{TRASPLANTE DE ESPECIES DE PECES COMO MECANISMO PRECURSOR DE INTRODUCCIÓN}

Según Gutiérrez-Bonilla et al. (2010), trasplantar especies es el movimiento de especies de una región a otra dentro del mismo país, y conlleva el riesgo de que se vuelvan invasoras. Biológicamente es válido pensar que si las especies nativas en algún momento de la historia estuvieron presentes en una región y ya no lo están, no puede suponerse que sea viable su reintroducción, pues las condiciones del equilibrio original no están presentes y los ecosistemas poseen readecuaciones y nuevos balances ecológicos que se deben conocer y valorar previamente (Alvarado-Forero y Gutiérrez-Bonilla,2002).Los trasplantes ofrecen los mismos problemas y peligros potenciales que la introducción (Lachner et al., 1970), pues son elementos adventicios, de los cuales no existe evidencia de que hubiesen estado representados en los ecosistemas receptores. Este hecho es importante pues cada ecosistema tiene una dinámica y equilibrio propio. Así, los elementos adventicios de la misma manera que eventualmente pueden ser un éxito como introducción, se pueden convertir en factores adversos de difícil mitigación.

Una vez producida la introducción de los peces, se abren las puertas para que se lleven a cabo los trasplantes de las especies hacia regiones diferentes a donde se produjo la introducción, debido a varias causas: 1) desconocimiento de las buenas prácticas de manejo de los estanques de cultivo, 2) uso de alevinos para el cultivo intensivo en estanques, 3) repoblamiento de embalses y ciénagas, por parte de entidades nacionales, regionales y locales. La mayor parte de los trasplantes de Colombia se han originado por la acuicultura, pues en la medida en que se han ido desarrollando los paquetes tecnológicos de algunas especies, se les ha comenzado a utilizar en regiones diferentes a sus cuencas de origen, bien sea por el transporte de parentales o por la compra de alevinos. Este comercio no está siendo controlado por las autoridades competentes, lo cual trae como consecuencia que la introducción de estas especies responda más al capricho de los acuicultores que a las necesidades reales de las regiones (Álvarez-León et al., 2002).

\section{ESTADO DE CONOCIMIENTO DE LAS ESPECIES DE PECES INTRODUCIDAS Y TRASPLANTADAS EN COLOMBIA}

La situación actual en nuestro país acrecienta el interés por el estudio de diferentes aspectos ecológicos de las especies no nativas fuera de su área de origen, ya que estas especies varían a diferentes escalas espaciales y temporales en sus procesos biológicos, lo que puede causar diversos impactos sobre el medio ambiente, sobre la economía regional e incluso sobre la sociedad (Gutiérrez-Bonilla, 2006; Prenda et al., 2006; Schwindt, 2007; Baptiste et al., 2010; Oviedo-Castro, 2012). Para Colombia, Welcomme $(1981,1998)$ hizo referencia a 10 especies de peces 
introducidas, posteriormente Hernández-Camacho (1984), Patiño (1973), Rodríguez-Gómez (1980, 1984), Rodríguez y Phelps (1982) y ÁlvarezLeón y Rodríguez-Forero (2000) registraron la introducción de 35 especies no nativas (29 ornamentales, 6de consumo), de las cuales 11 ya estaban en cuerpos de aguas naturales (6de consumo y 5ornamentales). COPESCAL (1986) y Alvarado-Forero y Gutiérrez-Bonilla (1999) confirmaron 49 especies, 26 ornamentales de origen asiático, que por su manejo han sido de difícil detección.

Hasta la fecha se tiene conocimiento de que los peces dulceacuícolas introducidos en Colombia ascienden a 81, provenientes de un amplio rango de distribución mundial y destinados principalmente a la acuicultura y a la ornamentación (Álvarez-León et al., 2002).Casos para asumir de manera inmediata en Colombia han sido reconocidos por el Ministerio de Ambiente, Vivienda y Desarrollo Territorial en 2008, que declaró oficialmente como especies invasoras a las especies:Oncorhynchus mykiss, Oreochromis niloticus, O. mossambicus, Cyprinus carpio, Micropterus salmoides y Trichogaster pectoralis (Gutiérrez-Bonilla et al., 2010). Además, se tenía conocimiento de al menos 23 trasplantes realizados por particulares y entidades gubernamentales sin que para ello se hubiesen llevado a cabo los estudios biológicos previos, que estableciesen el efecto de tales acciones según lo reportado por Álvarez-León et al. (2002). Posteriormente, Gutiérrez-Bonilla et al. (2010) registraron 55 trasplantes de especies para el país, siendo el Valle del Cauca en donde se registra el mayor número de especies introducidas y trasplantadas (90 especies), seguido por Antioquia (71 especies) y en tercer lugar Caldas (63 especies).

Sobre los efectos de algunas de estas especies en los ecosistemas acuáticos de Colombia, se pueden encontrar en los trabajos de Caraballo (2009), Anderson y Maldonado-Ocampo (2010), Baptiste et al. (2010), Galván-Guevara y De La Ossa (2011) y Gutiérrez-Bonilla et al. (2012). Este conocimiento todavía sigue siendo bajo con respecto al número de especies introducidas y al número de lugares en las que se reportan. Además, la complejidad que rodea las relaciones ecológicas entre introducidas-nativas o trasplantadas-nativas es muy elevada y debe ser desentrañada para poder arbitrar medidas correctoras que limiten las consecuencias adversas de las especies introducidas en los ecosistemas.

Recientemente, Gutiérrez-Bonilla(2012, p.264) propone recomendaciones y oportunidades para el control y manejo de las especies introducidas y trasplantadas, resaltando que ante todo dichas actividades deberán tener en cuenta:

[...] los análisis de lo pueda ocurrir con las diferentes actividades y practicar consulta científica obligada con el IIRBAvH, el INVEMAR, el SINCHI y el IIAP, según sea el caso y la región, para así generar coordinación y coherencia institucional, frente a la conservación de la diversidad biológica, razón de ser y obligación de los institutos de acuerdo al Decreto 1603 de 1994. Además, cuando de otorgar permisos para introducciones de flora, fauna, recursos hidrobiológicos, recursos pesqueros y microorganismos se trate, lo deseable sería que las Autoridades Ambientales estén involucradas en la toma de decisiones y si esta es aprobada, participen en las posteriores actividades de seguimiento. 


\section{ESTADO DE CONOCIMIENTO DE LAS ESPECIES DE PECES INTRODUCIDAS Y TRASPLANTADAS EN EL DEPARTAMENTO DE CALDAS, COLOMBIA}

Para el departamento de Caldas, Alvarado-Forero \& Gutiérrez-Bonilla (2002) mencionan 65 especies introducidas (63 peces, 2 crustáceos) de las cuales 46 peces son ornamentales de origen asiático, o para cultivos a pequeña y mediana escala, estando la mayoría de ellas en confinamiento (Alvarado-Forero \& Gutiérrez-Bonilla, 2002). Además CORPOCALDAS (1999) y Mojica-Corzo\&Galvis-Vergara(2000) registran en aguas naturales de varias microcuencas hidrográficas de todo el departamento especies como: Colossoma macropomum, Ctenopharyngodon idella, Hypophthalmichthys nobilis, Micropterus salmoides, Oreochromis mossambicus, O. niloticus niloticus, Oreochromis spp., Oncorhynchus mykiss, Piaractus brachypomus y Rasbora trilineata. También, mencionan 67 especies ornamentales confinadas en acuarios y para el uso de actividades comerciales en estanques piscícolas (Tabla 2). Así mismo, Serna- Mendoza y colaboradores (2010) resaltan la importancia del departamento de Caldas como pionero en la utilización de peces introducidos y con la técnica de biosístemas integrados.

Por otro lado, Gutiérrez-Bonilla et al. (2010) registraron 63 especies introducidas, la mayoría de las especies están presentes en cuerpos de agua artificiales, pero eventualmente se ha venido presentado su registro en aguas naturales, debido a los escapes o a las introducciones intencionales (Gutiérrez-Bonilla et al., 2010). Las especies introducidas más abundantes están representadas por las especies del género Oreochromis, le siguen las carpas del género Cyprinus, y como especies trasplantadas las de mayor registro son la cachama negra (C. macropomum) y la cachama blanca (P. brachypomus).

Tabla 1. Familias y número de especies por ordentaxonómico para los peces no nativos y trasplantados en el departamento de Caldas

Box 1. Families and number of species in taxonomic orders of non.natives fishes and trasplanted presents of the department of Caldas

\begin{tabular}{|ccc|}
\hline Orden / Orders & Familias / Families & \# Especies / \# Species \\
\hline Osteoglossiformes & Osteoglossidae & 2 \\
Characiformes & Prochilodontidae & 1 \\
& Characidae & 7 \\
Siluriformes & Loricariidae & 2 \\
& Pimelodidae & 1 \\
Salmoniformes & Salmonidae & 2 \\
Cypriniformes & Cyprinidae & 12 \\
Atheriniformes & Melanotaeniidae & 4 \\
Cyprinodontiformes & Poeciliidae & 8 \\
Perciformes & Symphysanodontidae & 1 \\
& Centrarchidae & 1 \\
& Cichlidae & 24 \\
& Osphronemidae & 7 \\
Total & $\mathbf{1 3}$ & 72 \\
\hline
\end{tabular}


Posteriormente, Restrepo-Santamaría y Álvarez-León (2011) ponen en evidencia 14 especies introducidas, 8 especies trasplantadas y 17 especies de peces utilizadas en piscicultura, siendo la familia Cichlidae la más representativa para la piscicultura del departamento (Tabla 1). Teniendo en cuenta que las especies trasplantadas ofrecen los mismos problemas y peligros potenciales que la introducción, y cualquier introducción con fines de cultivo es una adición potencial a la fauna silvestre, entonces están registrando en su investigación 24 especies no nativas de carácter invasor en los sistemas hídricos que surcan el departamento (RestrepoSantamaría\& Álvarez-León, 2011). Así mismo, registran en aguas naturales las especies: Carassius auratusauratus, Cichla monoculus, Colossoma macropomum, Cyprinus carpiocarpio, Oncorhynchus mykiss, Oreochromis mossambicus, O. niloticus niloticus, O. urolepis hornorum, Oreochromis spp., Piaractus brachypomus, Poecilia latipunctata, $P$. reticulata, Xiphophorus helleri y Zungaro zungaro (Restrepo-Santamaria y ÁlvarezLeón, 2011).

Dada la importancia de la introducción y el trasplante de especies ícticas y el papel fundamental que desempeñan sobre las especies nativas, profundizamos acerca de las especies de peces no nativas que se encuentran actualmente en esta región; a continuación ponemos en evidencia los resultados de este compendio de información acerca de la introducción y trasplante de especies no nativas. En este documento se registran 72 especies no nativas, la mayoría de las especies están presentes en cuerpos de agua artificiales, sin embargo 18 especies no nativas de carácter invasor se encuentran en aguas naturales de algunos ecosistemas hídricos que surcan el departamento (Tabla 2). Del total de especies 33 pertenecen al orden Perciformes, seguido de los Cypriniformes (12 especies), Characiformes (8 especies) y los Cyprinodontiformes (8 especies). Las familias con mayor número de especies corresponden en su orden: Cichlidae con 24, Cyprinidae con 12, Poeciliidae con 8 y Characidae con 7, las restantes familias tienen de 1 a 4 especies (Tabla 1).La mayoría de estas especies han sido simplemente registradas o tienen estudios con fines de investigación aplicada sobre el cultivo de peces a nivel agrícola y comercial, a fin de suministrar bases para un desarrollo racional de la piscicultura.

A continuación se describen las abreviaturas usadas para interpretar el listado taxonómico de las especies: especies no nativas (nnat), especies trasplantadas (tra), especie no nativa que se encuentra en aguas naturales (agn), especie no nativa que se encuentra en aguas artificiales (psc), especie no nativa que se halla en acuarios para comercialización (pdo). 
Tabla 2. Peces no nativos y trasplantados presentes en el departamento de Caldas. Fuentes: 1) Alvarado-Forero \& Gutiérrez-Bonilla (2002); 2) Gutiérrez-Bonilla et al. (2010); 3) Restrepo-Santamaría \& Alvarez-León (2011)

Box 2. These non native fish and translocations present of the Department of Caldas. Sources: 1) Alvarado-Forero \& Gutiérrez-Bonilla (2002); 2) Gutiérrez-Bonilla et al. (2010); 3) Restrepo-Santamaría \& Alvarez-León (2011)

\begin{tabular}{|c|c|c|c|}
\hline Taxón / Taxon & $\begin{array}{l}\text { Nombre Común } \\
\text { Common name }\end{array}$ & $\begin{array}{l}\text { Observaciones / } \\
\text { Observations }\end{array}$ & $\begin{array}{l}\text { Referencias } \\
\text { References }\end{array}$ \\
\hline \multicolumn{4}{|l|}{ Osteoglossiformes } \\
\hline \multicolumn{4}{|l|}{ Osteoglossidae } \\
\hline Arapaima gigas (Schinz, 1822) & Pirano, paiche, pirarucu & psctra & 3 \\
\hline $\begin{array}{l}\text { Osteoglossum bicirrhosum (Cwier, } \\
1829 \text { ) }\end{array}$ & Arawana & psc tra pdo & 1,2 \\
\hline \multicolumn{4}{|l|}{ Characiformes } \\
\hline \multicolumn{4}{|l|}{ Prochilodontidae } \\
\hline \begin{tabular}{|l|} 
Prochiodus \\
Steindachner, 1879
\end{tabular} & Bocachico, chupapata & nnat psc & $1,2,3$ \\
\hline \multicolumn{4}{|l|}{ Characidae } \\
\hline $\begin{array}{l}\text { Brycon amazonicus (Spix \& } \\
\text { Agassiz, 1829) }\end{array}$ & Yamu & psctra & 3 \\
\hline Brycon mooreisteindachner, 1878 & $\begin{array}{l}\text { Mueluda, sardinata, } \\
\text { dorada }\end{array}$ & psc & 3 \\
\hline Brycon sinuensis Dalh, 1955 & Sardinata, dorada & psc & 3 \\
\hline $\begin{array}{l}\text { Cheirodon interruptus (Jenyns, } \\
1842 \text { ) }\end{array}$ & Tetra del Congo & nnat psc & 1,2 \\
\hline $\begin{array}{l}\text { Colossoma macropomum (Cuvier, } \\
1816 \text { ) }\end{array}$ & Cachama negra & agn nnat tra & $1,2,3$ \\
\hline \begin{tabular}{|ll} 
Gymnocorymbus & ternetzi \\
(Boulenger, 1895) & \\
\end{tabular} & Monjita, black tetra & nnat psc & 1,2 \\
\hline $\begin{array}{l}\text { Hyphes sobrycon filammeus Myers, } \\
1924\end{array}$ & Tetra llama & nnat psc & 1,2 \\
\hline $\begin{array}{l}\text { Piaractus brachypomus (Cuvier, } \\
1818 \text { ) }\end{array}$ & $\begin{array}{l}\text { Cachama blanca, } \\
\text { morocoto, cachama roja, } \\
\text { pacu }\end{array}$ & agn nnat psc tra & $1,2,3$ \\
\hline \multicolumn{4}{|l|}{ Siluriformes } \\
\hline \multicolumn{4}{|l|}{ Loricariidae } \\
\hline $\begin{array}{l}\text { Hypostomus ventromaculatus } \\
\text { Boeseman, } 1968\end{array}$ & Cucha & psc tra & 3 \\
\hline $\begin{array}{l}\text { Wypostomus watwata Hancock, } \\
1828\end{array}$ & Cucha & psctra & 3 \\
\hline \multicolumn{4}{|l|}{ Pimelodidae } \\
\hline Zungaro zungaro (Humboldt, 1821) & $\begin{array}{ll}\text { Sapo, } & \text { pejesapo, } \\
\text { bagresapo } & \end{array}$ & agn nnat psc tra & 3 \\
\hline \multicolumn{4}{|l|}{ Salmoniformes } \\
\hline \multicolumn{4}{|l|}{ Salmonidae } \\
\hline $\begin{array}{l}\text { Oncorhynchus mykiss (Walbaum, } \\
1792 \text { ) }\end{array}$ & Trucha arco iris & agn nnat psc & $1,2,3$ \\
\hline Salvelinus fontinalis (Mitchill, 1814) & Trucha de arroyo & nnat psc & 1,2 \\
\hline \multicolumn{4}{|l|}{ Cypriniformes } \\
\hline \multicolumn{4}{|l|}{ Cyprinidae } \\
\hline $\begin{array}{l}\text { Carassius auratus } \\
\text { (Linnaeus, 1758) }\end{array}$ & $\begin{array}{l}\text { Bailarina, gold fish, } \\
\text { golfish calico, goldfish } \\
\text { sarasa, burbuja roja, } \\
\text { calico roja con blanca, } \\
\text { escama de perla roja, } \\
\text { telescopio }\end{array}$ & agn nnat & $1,2,3$ \\
\hline $\begin{array}{l}\text { Ctenopharyngodon } \\
\text { (Valenciennes, 1844) }\end{array}$ & $\begin{array}{l}\text { Carpa cabezona, carpa } \\
\text { herbivora }\end{array}$ & agn nnat & 1,2 \\
\hline $\begin{array}{l}\text { Cyprinus carpio carpio Linnaeus, } \\
1758 \\
\end{array}$ & Carpa comun & agn nnat psc & $1,2,3$ \\
\hline
\end{tabular}




\begin{tabular}{|c|c|c|c|}
\hline Lanio alboineatus (blyth, 1860) & Danıo perla & nnat psc & 1,2 \\
\hline Danio rerio (Hamilton, 1822) & Danio rerio & nnat psc & 1,2 \\
\hline $\begin{array}{l}\text { Epalzeorhynchos bicolor (Smith, } \\
\text { 1931) }\end{array}$ & Tiburon colirrojo & nnat psc & 1,2 \\
\hline $\begin{array}{l}\text { Hypophthaimichthys nobilis } \\
\text { (Richardson, 1845) }\end{array}$ & Carpa cabezona & agn nnat psc & 1,2 \\
\hline $\begin{array}{l}\text { Puntius conchonius (Hamilton, } \\
1822 \text { ) }\end{array}$ & Barbu rojo & nnat psc & 1,2 \\
\hline Puntius tetrazona (Bleeker, 1855) & Albina & nnat psc & 1,2 \\
\hline $\begin{array}{l}\text { Rasbora trilineata Steindachner, } \\
1870\end{array}$ & Rasbora tres lineas & agn nnat & 1,2 \\
\hline $\begin{array}{l}\text { Tanichthys albonubes Linnaeus, } \\
1932\end{array}$ & Neon chino & nnat psc & 1,2 \\
\hline $\begin{array}{l}\text { Trigonostigma } \\
\text { (Duncker, 1904) }\end{array}$ & Arlequin & nnat psc & 1,2 \\
\hline \multicolumn{4}{|l|}{ Atheriniformes } \\
\hline \multicolumn{4}{|l|}{ Melanotaeniidae } \\
\hline $\begin{array}{l}\text { Melanotaenia australís (Castelnau, } \\
\text { 1875) }\end{array}$ & & nnat psc & 1,2 \\
\hline $\begin{array}{l}\text { Melanotaenia boesemani Allen \& } \\
\text { Cross, } 1980\end{array}$ & Boeman's raimbowfish & nnat psc & 1,2 \\
\hline $\begin{array}{ll}\text { Melanotaenia } & \text { herbertaxelrodi } \\
\text { Allen, } 1981 & \end{array}$ & Lake Tebera rainbowfish & nnat psc & 1,2 \\
\hline $\begin{array}{l}\text { Melanotaenia splendida splendida } \\
\text { (Peters, 1866) }\end{array}$ & Eastern rainbow fish & nnat psc & 1 \\
\hline \multicolumn{4}{|l|}{ Cyprinodontiformes } \\
\hline \multicolumn{4}{|l|}{ Poeciliidae } \\
\hline Foecilia latioinna (Lesueur, 1821) & Molinesía de velo & nnat psc & $1,2,3$ \\
\hline Poecilia latipunctata Meek, 1904 & Pipon & agn nnat & 1,3 \\
\hline Poecilia reticulata Peters, 1859 & Guppy & agn nnat psc & $1,2,3$ \\
\hline $\begin{array}{l}\text { Poecila sphenops valenciennes, } \\
1846\end{array}$ & TMolly & nnat psc & 1,2 \\
\hline Poecilla velifera (Regan, 1914) & Molinesia de velo & nnat psc & 1,2 \\
\hline Xiphophorus helleri Heckel, 1848 & Espada, coliespada & agn nnat psc & $1,2,3$ \\
\hline $\begin{array}{l}\text { Xiphophorus maculatus (Günther, } \\
1866 \text { ) }\end{array}$ & Platy bandera & nnat psc & $1,2,3$ \\
\hline Xiphophorus variatus (IVeek, 1904) & Platy & nnat psc & $1,2,3$ \\
\hline \multicolumn{4}{|l|}{ Perciformes } \\
\hline \multicolumn{4}{|l|}{ Symphysanodontidae } \\
\hline $\begin{array}{l}\text { Symphysanodon aequifasciatus } \\
\text { Pellegrin, } 1904\end{array}$ & Disco azul & nnat psc & 1,2 \\
\hline \multicolumn{4}{|l|}{ Centrarchidae } \\
\hline $\begin{array}{l}\text { Wicropterus salmoides (Lacepede, } \\
1802 \text { ) }\end{array}$ & & agn nnat & 1,2 \\
\hline \multicolumn{4}{|l|}{ Cichlidae } \\
\hline $\begin{array}{l}\text { Amatitlania nigrofasciata (Günther, } \\
1867 \text { ) }\end{array}$ & Pez convicto & nnat psc & 1,2 \\
\hline $\begin{array}{l}\text { Amphiophus } \\
\text { (Günther, 1864) }\end{array}$ & & nnat psc & 2 \\
\hline $\begin{array}{l}\text { Astronotus ocellatus (Agassiz, } \\
\text { 1831) }\end{array}$ & Oscar & nnat psc & 1,2 \\
\hline $\begin{array}{l}\text { Caquetaia kraussï(Steindachner, } \\
\text { 1878) }\end{array}$ & Mojarra amarilla & $\mathrm{psc}$ & $1,2,3$ \\
\hline $\begin{array}{l}\text { Cichla ocellaris Bloch \& Schneider, } \\
1801\end{array}$ & Tucunare, pavon & agn nnat psc tra & $1,2,3$ \\
\hline Heros severus Heckel, 1840 & Falso escalar & nnat psc & 1,2 \\
\hline
\end{tabular}




\begin{tabular}{|c|c|c|c|}
\hline Maylandia zebra (Boulenger, 1899) & Zebra mbuna & nnat psc & 1,2 \\
\hline $\begin{array}{ll}\text { Welanochromis } & \text { auratus } \\
\text { (Boulenger, 1897) } & \end{array}$ & Golden mbuna & nnat psc & 1,2 \\
\hline $\begin{array}{l}\text { Melanochromis johannii (Eccles, } \\
\text { 1973) }\end{array}$ & Bluegray mbuna & nnat psc & 1,2 \\
\hline $\begin{array}{l}\text { Mikrogeophagus ramirezi (Myers \& } \\
\text { Harry, 1948) }\end{array}$ & Ramirezi & nnat psc & 1,2 \\
\hline $\begin{array}{l}\text { Neolamprologus brichardi (Poll, } \\
1974 \text { ) }\end{array}$ & Ciclio limon & nnat psc & 2 \\
\hline $\begin{array}{ll}\text { Oreochromis } & \text { aureus } \\
\text { (Steindachner, 1864) } & \\
\end{array}$ & Tilapia aurea, tilapia azul & nnat psc & $1,2,3$ \\
\hline $\begin{array}{l}\text { Oreochromis mossambicus } \\
\text { (Peters, 1852) }\end{array}$ & $\begin{array}{l}\text { Tilapia mosambica, } \\
\text { tilapia negra }\end{array}$ & agn nnat psc & $1,2,3$ \\
\hline $\begin{array}{l}\text { Oreochromis nioticus nioticus } \\
\text { (Linnaeus, 1758) }\end{array}$ & $\begin{array}{l}\text { Mojarra plateada, tilapia } \\
\text { plateada }\end{array}$ & agn nnat psc & $1,2,3$ \\
\hline Oreochromis spp. & Tilapia roja & agn nnat psc & $1,2,3$ \\
\hline $\begin{array}{l}\text { Oreochromis urolepis hornorum } \\
\text { (Trewavas, 1966) }\end{array}$ & Tilapia & agn nnat psc & $1,2,3$ \\
\hline $\begin{array}{ll}\text { Pelvicachromis } & \text { pulcher } \\
\text { (Boulenger, 1901) } & \end{array}$ & Rainbow krib & nnat psc & 1,2 \\
\hline $\begin{array}{l}\text { Pseudotropheus elongatus Fryer, } \\
1956\end{array}$ & Elongate mbuna & nnat psc & 1,2 \\
\hline $\begin{array}{l}\text { Pterophy"um scalare (Schultze, } \\
\text { 1823) }\end{array}$ & Pez escalar & nnat psc & 1,2 \\
\hline Rocio octofasciata (Regan, 1903) & Jack dempsey & nnat psc & 1,2 \\
\hline Tiapia rendalif (Boulenger, 1897) & Tilapia herbivora & nnat psc & $1,2,3$ \\
\hline Thorichthys meeki Brind, 1918 & Boca de fuego & nnat psc & 1 \\
\hline $\begin{array}{l}\text { Tropheops gracilior (Trewavas, } \\
\text { 1935) }\end{array}$ & Amarillo & nnat psc & 1 \\
\hline $\begin{array}{l}\text { Tropheops tropheops (Regan, } \\
1922 \text { ) }\end{array}$ & Tropheus dorado & nnat psc & 1,2 \\
\hline \multicolumn{4}{|l|}{ Osphronemidae } \\
\hline Betta splendens Regan, 1910 & Pezluchador & nnat psc & 1,2 \\
\hline Colisa lalia (Hamilton, 1822) & Colisa & nnat psc & 1,2 \\
\hline $\begin{array}{l}\text { Macropodus opercularis (Linnaeus, } \\
1758 \text { ) }\end{array}$ & Pez del paraiso & nnat psc & 1,2 \\
\hline Trichogaster leerii (Bleeker, 1852) & Gourami perla & nnat psc & 1,2 \\
\hline $\begin{array}{l}\text { Trichogaster microlepis (Günther, } \\
\text { 1861) }\end{array}$ & Plateado, luz de luna & nnat psc & 1,2 \\
\hline $\begin{array}{l}\text { Trichogaster pectoralis (Regan, } \\
1910 \text { ) }\end{array}$ & Gourami piel de culebra & nnat psc & 1,2 \\
\hline $\begin{array}{l}\text { Trichogaster trichopterus (Pallas, } \\
1770 \text { ) }\end{array}$ & Gourami tres puntos & nnat psc & 1,2 \\
\hline
\end{tabular}

\section{AGRADECIMIENTOS}

A Alejandra Correa-Bedoya, por su ayuda en el abstract, y a Luz Fernanda Jiménez-Segura por la lectura crítica del presente trabajo.

\section{BIBLIOGRAFÍA}

- Agostinho, A.A.;Pelicice, F.M. yJulio Jr, H.F. (2005). Introdução de espécies de peixes em águas continentais brasileiras: uma síntese. In: Rocha, O; Espindola, E.L.G.; Fenerich-Verani, N.; Verani, J.R. yRietzler, A.C. (eds.),Espécies Invasoras de Águas Doces: estudo 
de caso e propostas de manejo (pp.13-23). São Carlos:Editora da Universidade Federal de São Carlos.

- Allan, J.D. y Flecker, S. (1993). Biodiversity conservation in running waters: identifying the major factors that affect destruction of riverine species and ecosystems. BioScience, 43, 497-502.

- Alvarado-Forero, H. y Gutiérrez-Bonilla, F. de P. (1999). Especies hidrobiológicas continentales introducidas y trasplantadas y su distribución en Colombia. Inf. Técnico. Santa Fe de Bogotá, D.C.: Ministerio del Medio Ambiente, Instituto de Investigaciones de los Recursos Biológicos Alexander von Humboldt. 134 p.

- __. (2002). Especies hidrobiológicas continentales introducidas y trasplantadas y su distribución en Colombia. Bogotá: Ministerio del Medio Ambiente, Instituto de Investigación de Recursos Biológicos Alexander von Humboldt. 130p.+ 10 mapas.

- Álvarez-León, R.; Gutiérrez-Bonilla, F. de P.y Rodríguez-Forero, A.(2002).La introducción y trasplante de peces dulceacuícolas en Colombia: impactos ecológicos, económicos y legales.En: MojicaCorzo, J.ı.; Castellanos-Castillo, C.;Usma-Oviedo, J.S.y ÁlvarezLeón, R. (eds.),El libro rojo de los peces dulceacuícolas de Colombia. La Serie de Libros Rojos de Especies Amenazadas de Colombia (pp. 55-62 + 270-274).Santa Fe de Bogotá, D.C.: ICNUNC / IIRBAvH / MINAMBIENTE / Cl-Colombia.285 p.

- Álvarez-León, R. y Rodríguez-Forero, A. (2000). La acuicultura en Colombia: estado actual y perspectivas. Rev. INFOPESCA Internacional, 6, 40-47.

- Anderson, E.P. y Maldonado-Ocampo, J.A. (2010). A regional perspective on the diversity and conservation of tropical Andean fishes. Conservation Biology, 25(1), 30-39.

- Baptiste M.P.; Castaño, N.; Cárdenas, D.; Gutiérrez-Bonilla, F.P.; Gil, D.L. y Lasso-Alcalá, C.A. (eds.). (2010). Análisis de riesgo y propuesta de categorización de especies introducidas para Colombia. Bogotá, D.C.: Instituto de Investigación de Recursos Biológicos Alexander von Humboldt. 200 p.

- Begon, M.; Townsend, C.R. \& Harper, J.L. (2006). Ecology: From individuals to ecosystems. 4 ed. MA, USA: Blackwell Publishing. $746 \mathrm{p}$.

- Caraballo, P. (2009). Effect of tilapia Oreochromis niloticus over the fisheries in El Guájaro Reservoir Atlántico - Colombia. Rev. MVZ Córdoba 14(3): 1796-1802.

- COPESCAL. (1986). Introducción de especies ícticas y conservación de los recursos genéticos de América Latina y el Caribe. $O P, 3,1-$ 12. FAO, COPESCAL.

- CORPOCALDAS. (1999). Informe sobre la mortalidad de tilapias en la Cuenca del río Chinchiná. Manizales: Corporación Autónoma Regional de Caldas. 6 p.

- Courtenay, W.R.Jr. (1993). Biological pollution through fish introductions. En: Mcknight, B.N. (ed.), Proc. Symp. Biological Pollution: The Control and Impact of Invasive Exotic Species (pp. 36-61). Indianapolis (USA): Indiana Academy of Science.

- Cucherousset, J. y Olden J.D. (2011). Ecological Impacts of Nonnative Freshwater Fishes. Fisheries, 36 (5), 215-230.

- Delariva, R.L. y Agostinho, A.A. (1999). Introdução de espécies: uma síntese comentada. Acta Scientiarum, Maringá-PR, 21 (2), 255-262.

- Dudgeon, D.;Arthington, A.H.;Gessner, M.O.; Kawabata, Z.; Knowler, D.J.;Lévêque, C.L.; Naiman, R.J.; Prieur-Richard, A.; Soto, D.; Stiassny, M.L.J. ySullivan, C.A. (2006). Freshwater biodiversity: 
importance, threats, status and conservation challenges. Biological Reviews, 81, 163-182.

- Elvira, B. (2005). Peces invasores: una amenaza a escala mundial para la biodiversidad. Ciencia y Conservación: Trofeo Pesca, 136, 104-105.

- Elvira, B.; Almodóvar, A.; Nicola, G.G. y Almeida, D. (2007). Impacto de los peces y cangrejo introducidos en el Parque Nacional de Cabañeros, pp. 181-193. En: Ramírez, L. y B. Asensio (eds.), Proyectos de investigación en parques nacionales: 20032006. Madrid: Organismo Autónomo Parques Nacionales.

- Galván-Guevara, S y De la Ossa, J. (2011). Exotic fauna and transplanted fauna with more representativeness in Colombia. Rev. Colombiana Ciencias Animales, 3(1): 166-179.

- Gozlan, R. E. 2008. Introduction of non native freshwater fish: is it all bad? Fish and Fisheries, 9:106-115.

- Gozlan, R. E. y Newton, A. C. (2009). Biological invasions: benefits versus risks. Science, 324: 1015-1016.

- Gozlan, R.E.; Britton, J.R.; Cowx, I. \& Copp, G.H. (2010). Current knowledge on non-native freshwater fish introductions. Journal of Fish Biology, 76,751-786.

- Granado-Lorencio, C. (2002). Ecología de peces. Universidad de Sevilla. Secretariado de publicaciones. 352 pág.

- Gurevitch, J. y Padilla, D.K. (2004). Are invasive species a major cause of extinctions? Trends in Ecology \& Evolution, 19(9), 470474.

- Gutiérrez-Bonilla, F. de P. (2006). Estado de conocimiento de especies invasoras. Propuesta de lineamientos para el control de los impactos. Bogotá, D.C.: Instituto de Investigación de Recursos Biológicos Alexander von Humboldt. 156 p.

- _ (2012). Recomendaciones y oportunidades para el control y manejo de las especies introducidas y trasplantadas. Capítulo 6. En: Gutiérrez-Bonilla, F. de P.; Lasso-Alcalá, C.A.; Baptiste, M.P.; Sánchez-Duarte, P. y Díaz, A.M. (eds.), VI Catálogo de la biodiversidad acuática exótica y trasplantada en Colombia: moluscos, crustáceos, peces, anfibios, reptiles y aves (pp. 263272). Serie editorial Recursos Hidrobiológicos y Pesqueros Continentales de Colombia. Bogotá, D.C.: Instituto de investigación de los recursos biológicos Alexander von Humboldt (IAvH). 345 p.

- Gutiérrez-Bonilla, F de P.; Lasso-Alcalá, C.A.; Baptiste, M.P.; Sánchez-Duarte, P. y Díaz, A.M. (2012). VI Catálogo de la biodiversidad acuática exótica y trasplantada en Colombia: moluscos, crustáceos, peces, anfibios, reptiles y aves. Serie editorial Recursos Hidrobiológicos y Pesqueros Continentales de Colombia. Bogotá, D.C.: Instituto de investigación de los recursos biológicos Alexander von Humboldt (IAvH). 335 p.

- Gutiérrez-Bonilla, F. de P.; F.; Lasso-Alcalá, C.A.; Sánchez-Duarte, P. y Gil, D.L. (2010). Análisis de riesgo para especies acuáticas continentales y marinas. En: Baptiste M.P.; Castaño, N.; Cárdenas, D.; Gutiérrez, F.P.; Gil D.L. y Lasso, C.A. (eds.), Análisis de riesgo y propuesta de categorización de especies introducidas para Colombia. Bogotá, D.C.: Instituto de Investigación de Recursos Biológicos Alexander von Humboldt, 335 p.

- Hernández-Camacho, J.I. (1984). Vistazo general sobre la protección de la naturaleza en Colombia. Bogotá, D.E.: INDERENA. Inédito.

- IUCN -International Union for Conservation of Nature-. (2009). Extinction crisis continue sapace. Disponible en: web: 
http://www.iucn.org/?4143/Extinction-crisis-continues-apace [acceso 1 de abril de 2013].

- Lachner, E.A.; Robins, C.R.y Courtenay, W.R. (1970). Exotics fishes and other aquatic organisms introduced into North America. Smithsonian Contrib. Zool., 59, 1-29.

- Lodge, D.M. (1993). Biological invasions: lessons for ecology. Trends in Ecology \& Evolution, 8, 133-137.

- Mojica-Corzo, J.I. y Galvis-Vergara, G. (2000). Revisión, valoración y actualización de los estudios ícticos de las obras de transvase bajo del río Guarinó. HIDROMIEL. Inf. Técnico. Bogotá D.C. 33 p.

- Moyle, P.B. y Leidy, R.L. (1992). Loss of biodiversity in aquatic ecosystems: evidence from fish faunas. In: Fiedler, P.I. \& Jain, S.K. (eds.),Conservation biology: The theory and practice of nature conservation, preservation and management (pp. 127-169). New York (NY) USA: Chapman and Hall.

- Moyle, P.B. y Light, T. (1996). Biological invasions of fresh water: empirical rules and assembly theory. Biological Conservation, 78, 149-162.

- Oviedo-Castro, J. M. 2012. Diagnóstico socio-económico de la pesca continental y artesanal en el Departamento de Caldas - 2011. INCODER. Manizales (Caldas). ). Inf. Técnico Final, 31 p.

- Patiño, A. (1973). Guía práctica de la acuicultura artesanal. Cali: ANPAC.

- PNGIBSE. (2012). Política Nacional para la Gestión Integral de la Biodiversidad y los Servicios Ecosistémicos. Bogotá, D.C.: Ministerio de Medio Ambiente y Desarrollo Sostenible. 133 p.

- Prenda, J.; Blanco-Garrido, F.; Hermoso, V.; Clavero, M. y Menor, A. (2006). ¿Son realmente importantes las especies exóticas en la conservación de los ríos ibéricos? El caso de los peces. $\mathrm{V}$ Congreso Ibérico de Gestión y Planificación del Agua (Cuencas Compartidas, Claves para la Gestión Sostenible del Agua y del Territorio). Congreso Ibérico de Gestión y Planificación del Agua, Núm. 5. Faro (Portugal). Fundação Nova Cultura da Água. Universidade Do Algerve. p. 4-5.

- Restrepo-Santamaría, D. y Álvarez-León, R. (2011). Peces del Departamento de Caldas, Colombia. IIRBAvH-Rev. Biota Colombiana, 12 (1), 95-112.

- Rodríguez, D. yPhelps, R. (1982). Determinación del efecto de la mojarra africana Tilapia nilotica sobre una población de 13 especies de peces nativas del área del Bajo Magdalena. INDERENA - Rev. Divulg. Pesq., 22 (1), 1-26.

- Rodríguez-Gómez, H. (1980).Peces exóticos introducidos a Colombia. Mem. III Simp. Lat.-Amer. y IV Sem. Colombiano de Acuicultura. Cartagena (Bol.), Colombia, agosto 25-29.p. 1-26.

- . (1984). Peces exóticos introducidos y establecidos en aguas colombianas. Bogotá, D.E.: INDERENA. 36 p.

- Sarmiento, F.O. (2000). Diccionario de Ecología: Paisajes, Conservación y Desarrollo Sustentable para Latinoamérica. UNU / CLACS / NSF / AMA / CAF / ABYA-YALA / CEPEIGE. Quito: Editorial Abya-Yala. $226 \mathrm{p}$.

- Schwindt, E. (2007). Especies introducidas. Informe Técnico para la publicación en internet y en formato digital. Proyecto Atlas de Sensibilidad Ambiental de la costa y el Mar Argentino, D Boltovskoy (ed.). http://www.atlas.ambiente.gov.ar

- Serna-Mendoza, C. A., M. Salazar de Cardona, N. RodríguezValencia y R. Álvarez-León. 2010. Biosistemas integrados y sus 
interrelaciones con el desarrollo sostenible y el desarrollo humano y social. Univ. de Manizales - Centro de Investigaciones en Medio Ambiente y Desarrollo (CIMAD) - Maestría en Desarrollo Sostenible y Medio Ambiente / Corporación Autónoma Regional de Caldas (CORPOCALDAS). Manizales (Caldas) Colombia, 109 p.

- Simberloff, D. (1996). Hybridization between native and introduced wildlife species. Importance for Conservation.Wildlife Biology, 2, 143-150.

- Urano, K. (2006). Various ecological problems and biological invasion. In: Koike, F.; Clout, M.N.;Kawamichi, M.; De Poorter, M. \&lwatsuki, K. (eds.), Assessment and Control of Biological Invasion Risks. Published by Shoukadoh Book Sellers, Kyoto, Japan and the World Conservation Union (IUCN), Gland (Switzerland). 216 p.

- Welcomme, R.L. (1981). Registro de transferencias internacionales de especies de peces de aguas continentales. FAO Doc. Tec. Pes., 213, 1-20.

- $\quad$ (1988). International introductions of island aquatic species. FAO Fish. Tec. Pap., 294, 1-318.

1. Grupo de Ictiología Universidad de Antioquia, Instituto de Biología, Calle 67 No. 53-108, Laboratorio 7-309, Medellín, Antioquia. dasanta24@gmail.com

2. Fundación Verdes Horizontes. Manizales, Caldas, Colombia. ricardoalvarezleon@gmail.com

Para citar este artículo: Restrepo-Santamaría, D. \& Álvarez-León, R. (2013). Algunos aspectos sobre la introducción de especies, y estado del conocimiento sobre los peces introducidos en el departamento de Caldas, Colombia. Revista Luna Azul, 37, 268-281. Recuperado de http://lunazul.ucaldas.edu.co/index.php?option=content\&task=view\&id=855 